RESEARCH

\title{
Ambulation of patients who are mechanically ventilated: nurses' views
}

Curtis L, Irwin J (2017) Ambulation of patients who are mechanically ventilated: nurses' views. Nursing Management.

Date of submission: 8 November 2016; date of acceptance: 21 March 2017. doi: 10.7748/nm2017.e1599 Lee Curtis

Senior lecturer, Buckinghamshire New University, Uxbridge, UK

Julie Irwin

Academic enhancement manager, Buckinghamshire New University, Uxbridge, UK

Correspondence

lee.curtis@bucks.ac.uk

Conflict of interest

None declared

Peer review

This article has been subject to external double-blind peer review and checked for plagiarism using automated software

\begin{abstract}
Aims Equipment and skills in intensive care have advanced dramatically, and early rehabilitation and ambulation for patients in intensive care units (ICUs) is part of their journey to recovery. The aim of this study is to increase understanding of nurses' perspectives on ambulating mechanically ventilated patients, and to determine why this is not a routine part of ICU patient care.

Method An interpretative phenomenological analysis method was used to extract data from semistructured interviews. The questions were piloted twice before being used in the main study.

Results Results identified two overarching themes, staff anxiety and organisational culture, within which there are several subthemes. The study also found that education and training programmes could increase staff confidence, and consequently result in routine ambulation of mechanically ventilated patients.

Conclusion The study identified that nursing staff are aware of the benefits of ambulation for patients in ICUs, but the personal satisfaction gained from undertaking this activity does not outweigh the anxiety it causes. This is compounded by the organisational culture of ICUs, for example, the hierarchical pyramid of leadership, which dictates that consultants decide when patients are ready to ambulate.

ambulation, intensive care unit, mechanical ventilation, nurses, nursing management
\end{abstract}




\section{Introduction}

Early rehabilitation of patients in intensive care units (ICUs) is a valid and necessary part of recovery, but identifying those who are suitable, or ready, to begin exercise must be based on physiological and psychological parameters to ensure the chosen technique is safe and feasible. Therefore, a comprehensive patient assessment must be undertaken first, to determine whether the physical and psychological benefits outweigh potential risks.

\section{Literature review}

Over the past two decades, there has been a significant increase globally in the level of clinical specialty and technology in ICUs (Zomorodi et al 2012, Vincent 2013). Alongside this, mortality rates have greatly reduced (Morris and Herridge 2007), with some authors reporting survival rates of patients admitted to ICUs at more than $80 \%$ (Drenan et al 2010). According to the National Institute for Health and Care Excellence (NICE) (2009), the goal of ICUs, which was simply to ensure patients survived until they were discharged to the wards, needs to be closely scrutinised, while Elliot et al (2011) suggested that ICU admission should be regarded as only a small part of patients' journeys on a continuum of critical illness recovery.

Ambulation is beneficial for patients in ICUs in terms of physical health, long-term psychological function and health-related quality of life (Hodgin et al 2009, Berney et al 2012), but despite evidence that suggests it is safe and feasible (Burtin et al 2009, Schweickert et al 2009), it is not universally implemented (Adler and Malone 2012, Berney et al 2012, Leditschke et al 2012). To increase knowledge about this topic, nurses responsible for this activity, as part of their care of patients in ICUs, were interviewed.

\section{Study aim}

The aim of the study is to understand ICU nursing staff's experience of ambulating patients who are mechanically ventilated.

\section{Method}

The methodology used for this qualitative study was interpretative phenomenological analysis (IPA), a case-based idiographic approach that attempts to emphasise, capture and interpret the lived experience of those studied (Fade 2004, Smith 2004). Although a relatively new research method based in psychology, IPA has gained a considerable following in recent years and is increasingly used in medicalbased research.

\section{Sample}

All band 5, 6, and 7 nurses who work with ventilated patients in the ICU of a London hospital, around 60 members of staff, were eligible for inclusion. Inclusion criteria were nurses employed by the trust, 
and actively involved in the care of mechanically ventilated patients. Recruitment was by return of an introductory letter sent to eligible participants. The respondents' names were separated into their banding, and placed in individual envelopes with a matching number to the band written on the exterior.

A blinded selection process was used, where a member of staff, who was not attached to the research, chose one numbered envelope from each group. This allowed more participants to be randomly recruited until data saturation occurred. The final sample was eight participants: three band 7 , three band 6 and two band 5 nurses.

\section{Data collection tools and methods}

Semi-structured interviews were used for data collection (Table 1), which enabled development of discussions to enhance understanding of the activity from each participant's perspective. Interviews were piloted twice with agency staff who had worked in the ICU for more than five shifts. This meant the interview questions and structure were tested on nurses with the same experiences as the study recruits, but who were not eligible for inclusion.

\section{Table 1. Semi-structured interview questions}

\begin{tabular}{|c|c|}
\hline \multicolumn{2}{|c|}{ Semi-structured interview questions } \\
\hline 1 & How long have you been working as a nurse in an intensive care unit setting? \\
\hline 2 & $\begin{array}{l}\text { As you have put yourself forward to participate in this research, I assume you } \\
\text { have some understanding of ambulating mechanically ventilated patients. Is } \\
\text { this correct? }\end{array}$ \\
\hline 3 & What are your thoughts on this activity? \\
\hline 4 & $\begin{array}{l}\text { If you have been actively involved in this activity, how did it make you feel? } \\
\text { If you have read or heard about this activity, how do you think you would } \\
\text { feel if you were asked to assist? }\end{array}$ \\
\hline 5 & What are your thoughts on the benefits and risks of this activity? \\
\hline 6 & Is this activity used as often as it could or should be? \\
\hline 7 & $\begin{array}{l}\text { In your opinion, who should be responsible for deciding when patients are } \\
\text { ready to participate in this activity? }\end{array}$ \\
\hline
\end{tabular}




\begin{tabular}{|l|l|}
\hline 8 & $\begin{array}{l}\text { If you could make changes to ensure this activity was performed more } \\
\text { frequently, what would they be? }\end{array}$ \\
\hline
\end{tabular}

\section{Data analysis}

The primary researcher transcribed the recordings after each interview before undertaking the next one. Transcribing and interviewing helped to develop a more 'personal' reproduction, allowing the 'essence' of the discussion to be captured (Bailey 2008), and was beneficial to the researcher, as it supported a natural interview skill to evolve based on continuing knowledge acquisition.

\section{Ethical and research approval}

Ethical approval was sought through the Integrated Research Application System (IRAS), and approved by the university's and hospital's research and development departments. To ensure anonymity, all participants' quotes were assigned a male gender pronoun, and direct quotes that could identify specific roles were altered to make them generic, however every attempt was made to preserve the original meaning.

\section{Results}

Ambulating mechanically ventilated patients was not classed as 'routine ICU practice' when the study was conducted, but participants were aware of the associated physical and psychological benefits. Participants with experience of this activity felt an element of satisfaction from their involvement: 'It sort of empowers you as a nurse as well, if you are actively involved in it.'

Two superordinate themes were identified, staff anxiety and organisational culture, within which several subthemes emerged (Table 2), and these are discussed below.

Table 2:. Main and subthemes drawn from the data

\begin{tabular}{|l|l|}
\hline Theme & Subthemes \\
\hline Staff anxiety & $\begin{array}{l}\text { Patient safety } \\
\text { Guidance on ambulation } \\
\text { Staff competence to undertake ambulation } \\
\text { Equipment }\end{array}$ \\
\hline Organisational culture & $\begin{array}{l}\text { Leadership } \\
\text { Staffing levels }\end{array}$ \\
\hline
\end{tabular}


\begin{tabular}{|l|l|}
\hline & \\
\hline
\end{tabular}

\section{Staff anxiety}

Participants' demonstrated anxiety about ambulation while discussing the topic. Anxiety is a prospective emotion, and the feelings projected emerge from people's subconscious, based on a fear of what 'could' happen.

\section{Patient safety}

None of the participants related this fear to their personal safety, instead they were concerned about their patients' safety. Their concerns were far ranging, and encompassed various potential adverse events that could negatively affect patients' health:

'They may collapse on you when they get up and walk them around, failure of equipment'.

'At risk of taking out of the patient, detaching from the patient, yes, mostly that one, and the stability of the patient'.

'So, anything can happen, basically if you ambulate them'.

'Have you assessed the patient thoroughly enough? Is the patient really stable, and is this patient really ready?'

'I think nurses should be more proactive in rehabilitation of the patient as well, but I think most of us are, like, quite scared to ambulate the patient on a ventilator'.

Their anxieties, and fear for their patients' safety, were genuine emotional responses. Nauert (2007) related this indirect conditioning, between anxiety and fear, to a type of 'social conditioning', where the spoken or visual description of an adverse event is judged to be an issue for the person's future wellbeing. This conditioning evokes an empathetic and reproducible response, which can lead to omission of the activity in the future. Reports of adverse incidents associated with ambulating mechanically ventilated patients are very low (Truong et al 2009), which reinforces the idea that participants' anxieties are based on a conditioned belief that something might go wrong, rather than facts.

\section{Guidance on ambulation}

Guidelines for mobilising critically ill patients, based on a series of parameters, would support evaluation of their suitability for ambulation. There are guidelines that support ICU practitioners to evaluate patients' suitability for mobilisation (Stiller 2007), and to identify barriers to active mobilisation (Leditschke et al 2012). Participants suggested that the introduction of guidelines would help identify who to mobilise, this making the decision-making process easier, and some indicated that they would feel more confident if guidelines were used:

'If the assessment has been done, and the patient is fit for such an activity, then any qualified nurse should be able to...', 
'Maybe we don't have that guideline, I feel, probably once we have that in place, I think we'll be able to mobilise (patients) with a bit more confidence'.

ICU nurses are highly trained, and regularly undertake interventions guided by their assessment of patients' stability or abilities. Participants mentioned a range of activities they undertake, including 'regular position changing', 'full body washing', and 'sitting patients out', which involve a level of passive or active mobility. Carrying out these tasks requires nurses to make comprehensive risk assessments, based on similar parameters to ambulation guidelines.

\section{Staff competence}

Defence mechanisms are part of everyday life, and prevent us from being subjected to overwhelming emotional insults, ideas and realities (Freud 1894), and our subconscious systems work continuously to protect our egos from these perceived anxieties. Bennett and Holmes (1975) suggested that we should direct unwanted, subjective, anxieties away from ourselves and ascribe them to other people, or to the activity itself:

'When I know what to do and I have proper guidance/training, something in place, I would be confident to take the patient', 'If we have the correct team, it can be safely managed'.

Competence equates to confidence (Blum et al 2010), a state of mind that can only be accessed through exposure to activities. The Critical Care Networks National Nurse Leads recognises this in its National Competency Framework, which suggests that competency encompasses a combination of skills, attitudes, values and technical abilities, as well as theoretical knowledge (Kynaston 2012). Lemire (2013) suggested that to become a 'master' in any field requires 10,000 hours of practice experience, therefore the importance of 'activity experiences' should kept in mind when developing staff competence.

\section{Equipment}

Participants' anxieties about equipment mainly related to disconnection and failure, rather than the type used. These problems can occur, but are described by Truong et al (2009) as easily rectified 'minor adverse events'. Whether these 'minor adverse effects' were perceived as more major in relation to patients' dependence on equipment is not known.

Interestingly, the participants in this study noted that their patients were often due for discharge, which is generally associated with low dependency levels:

'If the patient's on CPAP already we're, like, aiming to extubate on that so we don't usually get to mobilise them'.

\section{Organisational culture}

Guidet and Gonzalez-Roma (2011) identified that values and behaviours are shaped by the shared customs and beliefs held by employees in organisations. The participants in this study work as a group 
within a set of written, and unwritten, rules, developed over time, and this has led to acceptance of standardised practice.

Leadership

This theme overlaps with 'guidance on ambulation', but was considered an independent entity as the participants raised it as a separate topic. Most participants wanted someone else lead on the activity, particularly the ICU medical consultant:

'I definitely don't think that the nurses... It's either the physio, or the doctors, probably the consultant, that's what I think'.

'They always initiate the discussion (physio), so I think they, primarily, they could take the lead'.

'Ultimately, it's the decision of the consultant for anything that happens in ICU'.

'The nurse in charge would probably have a big say in the priority decision of the activity'.

Senior nurse participants said that other professionals deciding what their patients could do removes their decision-making role and effectively disempowers them. They indicated that constraints on staff decision making could be counterproductive, reduce engagement, promote disassociation from the activity, and ultimately lead to de-skilling:

'We teach them that, you know, nursing one to one, that is your patient for the day, but then someone comes along and says "right, we're ambulating", so sort of taking it out of their hands', 'You are nursing one to one, and then someone comes along and takes that decision out of your hands'.

The loss of intrinsic and extrinsic control is associated with increased stress and anxiety (Mclntosh and Spilka 1990), so maintaining some aspect of patient 'ownership' is essential for staff engagement, prevents skill regression, and could support acceptance of ambulation as a routine activity. Staffing levels

The image evoked by the participants was of a unit unable to support or accommodate ambulation routinely:

'ITU is quite busy so you don't have the staff there with you all the time, so you have to prioritise what time, make all the people aware that we're doing ambulation at this time... so sometimes when there's like things going around unexpectedly it kind of ruins all the plans.'

There appeared to be a subconscious belief that the activity is labour intensive, and participants did not seem to consider the multidisciplinary team's contribution, despite knowing that the decision to ambulate is made by the whole team:

'The only anxiety I have is the amount of people it involves, if the unit was busy it could sort of pull on our resources.'

These perspectives could be linked to the fact that ambulation is not routinely observed in the unit. Unusual activities generally lead to those who are not directly involved congregating to observe them 
(Lyell and Becker 2005), but because they are unusual this can trigger emotional feelings of stress in the observers.

Panksepp (2010) acknowledges that emotions are central to people's understanding of experiences, and dictate their engagement within the world. According to Greenglass and Burke (2000), when people are faced with the thought of undertaking an activity that involves a perceived greater workload, the default response is anxiety and pressure, and to stop doing it:

I have to say, it takes time to do that procedure (ambulation), and I have to mention the staff, there is not enough to support it', 'It's a difficult thing to balance, so you have to consider everything, although we want to do it the capacity to do it is more difficult'.

When this response was explored in more depth, one participant recollected an event and altered their reply:

'Having said that, it was all organised so that you actually only used the nursing staff in the bed spaces, so it probably wouldn't pull on our resources at the end of the day'.

The reality of the event was different from the perception.

Subconscious understanding of activities can be corrupted by conscious discussion, during which false information is innocently distributed. Newell and Shanks (2014) suggested that unconscious memories, or implicit biases, can influence people's conscious minds to motivate actions and decisions, while Brennan (2013) considered the psychological reinforcement of what is then 'believed', and perpetuated through groups, becomes the endemic culture, or 'group position'. This can prevent activities from being performed, or even considered, by the group.

\section{Limitations}

The study aimed to increase understanding of nursing staff's perceptions of ambulation of ICU patients, and to gain more insight into why the uptake of this activity is slow. However, the results only account for the participants' perceptions at one point in their history. According to Smythe et al (2008), experiences are continually in flux, are unique to individuals, and based in their own settings. Therefore, the findings cannot be generalised.

\section{Discussion}

Participants are aware that ambulation is beneficial to their patients, but organisational culture means that they appear to accept 'staffing levels' as a valid reason for not undertaking the activity. These perceptions can only be altered through evidence-based education that explains the risks to, and benefits for, patients, and the number of nurses required to undertake the task.

Staffing levels in ICUs are widely discussed in the literature (Royal College of Nursing 2003, Bray et al 2010, Francis 2010), and research suggests there are links between staffing levels and patient safety and outcomes (West et al 2002, Coombs and Lattimer 2007). Future studies should examine staffing levels in 
relation to ambulation of mechanically ventilated patients, and link the findings to the numerous studies on the benefits of this intervention (Stiller 2007, Hodgin et al 2009, Perme and Chandreshaker 2009, Truong et al 2009, Berney et al 2012).

New activities must be accepted by staff consciously and subconsciously, and this can only be achieved if a manageable level of stress is maintained during implementation. Anxiety is a complex issue that comes from a position of limited knowledge and understanding, and was identified in all subthemes. Benson and Dundis (2003) suggested that activities only become routine when they promote a feeling of 'internal comfort', which can only be felt when certain essential components are satisfied.

Glazer and Gyurak (2008) examined sources of occupational stress, and identified three areas that need to be satisfied to reduce nurses' anxieties, appreciation of the task to be performed, the type of patient who is going to perform the task, and the skill set of the staff involved. Similarly, Goodwin et al (2007) identified the need for nurses to be 'prepared', and to 'know that their patients would be safe' to reduce their anxieties. The participants in this study expressed anxieties related to all these areas.

Sports research suggests that performance and motivation are negatively affected by anxiety (Mudimela 2010), while Berridge and Kringelbach (2008) identified that changes to participants' patterns of behaviour are linked to their performance in an activity. The subconscious defence mechanism stores situations internally that it perceives to be too stressful, then externally exhibits them as distorted 'facts' (McLeod 2009). This could explain why the participants justified not undertaking the activity by suggesting it was 'too risky', or there were 'not enough staff'.

\section{Implications and recommendations for practice}

This study complements quantitative research conducted on this topic, that suggests that ambulating mechanically ventilated patients is beneficial for their physical and non-physical rehabilitation. It is safe to perform, and has short- and long-term benefits for patients, yet it is not routinely undertaken.

The study also adds to the knowledge and understanding about staff anxiety and organisational culture. Staff education could reduce the cycle of anxiety that is cultivated in ICUs, and lead to changes in the organisational culture of these environments.

A comprehensive training programme is required to reduce nurses' anxiety, and increase the use of ambulation in ICUs. Training should be educational and participatory, so that repeated exposure enables the subconscious to recognise the reality of the activity, and to promote discussions based on facts rather than perceptions. Education programmes should also include data about adverse incident levels, and patient outcomes.

There was much discussion about guidelines on ambulation during the interviews, but it is questionable whether these alone would increase use of this intervention, given the other issues related 
to low uptake. However, if guidelines are introduced they should include a parameter checklist that enables nurses to assess patients' suitability for ambulation. Senior nurses could encourage staff to complete the guidelines daily, to identify patients who are ready to ambulate. This would create an ICU in which senior staff take the lead on patient ambulation, and less senior staff develop their patientrelated decision-making skills.

Exposure to the activity can be gained through multidisciplinary simulation sessions, where the risk is low and the reward is high. This approach will help to manage nurses' stress and anxiety levels, ensure appropriate use of the multidisciplinary team's skills, and illustrate that ambulation does not increase nurses' workload. Patient ambulation can then be used in ICU, and staff will maintain and develop their skills appropriately, aligned to their roles and responsibilities.

\section{Conclusion}

Ambulation of mechanically ventilated patients is a treatment modality with short- and long-term benefits for patients, and can decrease financial costs by reducing the length of overall hospital stay, readmission rates, and primary care resources (NICE 2009). Early introduction of ICU rehabilitation, based on appropriate patient selection, is safe and feasible.

The two independent, but interrelated, superordinate themes identified by this study, staff anxiety and organisational culture, demonstrate staff perceptions, and identify the physical and psychological obstacles that they perceive restricts implementation of the activity. The study has helped to identify that although nurses understand the benefits associated with ambulation, they cannot accept it as routine practice until they feel comfortable and competent to undertake it. Yet, their confidence will only increase when it becomes a regular part of routine care.

The study also highlights that most of the participants' concerns transcended bands and levels of experience, which indicates that the core themes are pertinent to all.

A comprehensive education programme will help nurses feel more confident about ambulating mechanically ventilated patients, and their confidence will increase further when the activity becomes commonplace, which in turn will decrease their anxiety. It is only then that a paradigm shift can take place in ICUs, and ensure ambulation becomes routine practice.

Adler J, Malone D (2012) Early mobilization in the intensive care unit: a systematic review. Cardiopulmonary Physical Therapy. $23,1,5-13$. Bailey J (2008) First steps in qualitative data analysis: transcribing. Family Practice. 25, 2, 127-131.

Bennet D, Holmes D (1975) Influence of denial (situation redefinition) and projection on anxiety associated with threat to self-esteem. Journal of Personality and Social Psychology. 32, 5, 915-921.

Benson S, Dundis S (2003) Understanding and motivating health care employees: integrating Maslow's hierarchy of needs, training and technology. Journal of Nursing Management. 11, 5, 315-320.

Berney S, Haines K, Skinner E et al (2012) Safety and feasibility of an exercise prescription approach to rehabilitation across the continuum of care for survivors of critical illness. Physical Therapy. 92, 12, 1524-1535.

Berridge K, Kringelbach M (2008) Affective neuroscience of pleasure: reward in humans and animals. Psychopharmacology. 199, 457-480. 
Blum C, Borglund S, Parcells D (2010) High-fidelity nursing simulation: impact on student self-confidence and clinical competence. International Journal of Nursing Education Scholarship. 7, 1, 1-14.

Bray K, Wren I, Baldwin A et al (2010) Standards for Nurse Staffing in Critical Care Units.

www.ics.ac.uk/professional/standards_safety_quality/standards_and_guidelines/nurse_staffing_in_critical_care_2009 (Last accessed: 17 February 2013.)

Brennan N (2013) Behavioural and psychological influences on boards. Keeping Good Companies. 65, 8, 457-461.

Burtin C, Clerckx B, Robbeets C et al (2009) Early exercise in critically ill patients enhances short-term functional recovery. Critical Care Medicine. 37, 9, 2499-2505.

Coombs M, Lattimer V (2007) Safety, effectiveness, and costs of different models of organising care for critically ill patients: literature review. International Journal of Nursing Studies. 44, 1, 115-129.

Drennan K, Hicks P, Hart G (2010) Intensive Care Safety and Quality Survey: Australia and New Zealand 2007/2008. www.anzics.com.au/core/reports (Last accessed: 17 February 2013.)

Elliott D, McKinley S, Alison J et al (2011) Health-related quality of life and physical recovery after a critical illness: a multi-centre randomised controlled trial of a home-based physical rehabilitation program. Critical Care. 15, 3, 1-10.

Fade S (2004) Using interpretative phenomenological analysis for public health nutrition and dietetic research: a practical guide. Proceedings of the Nutritional Society. 63, 4, 647-653.

Francis R (2010) Independent Inquiry into Care Provided by Mid Staffordshire NHS Foundation Trust January 2005-March 2009 (Volumes I and 2). www.midstaffsinquiry.com/assets/docs/Inquiry Report-Vol1.pdf www.midstaffsinquiry.com/assets/docs/Inquiry Report-Vol2.pdf (Last accessed: 17 April 2013.)

Freud S (1894) The Neuro-Psychoses of Defense. In The Standard Edition of the Complete Psychological Works of Sigmund Freud. Volume 3. Hogarth Press, London.

Glazer S, Gyurak A (2008) Sources of occupational stress among nurses in five countries. International Journal of Intercultural Relations. 32, 1, 49-66.

Goodwin M, Sener I, Steiner S (2007) A novel theory for nursing education: holistic comfort. Journal of Holistic Nursing. 25, 4, 278-285.

Greenglass E, Burke R (2000) Hospital downsizing, individual resources, and occupational stressors in nurses. Anxiety, Stress and Coping: An International Journal. 13, 4, 371-390.

Guidet B, González-Romá V (2011) Climate and cultural aspects in intensive care units. Critical Care. 15, 6, 312.

Hodgin K, Nordon-Craft A, McFann K et al (2009) Physical therapy utilization in intensive care units: results from a national survey. Critical Care Medicine. 37, 2, 561-567.

Kynaston M (2012) National Competency Framework for Critical Care Nurses: Providing Registered Nurses with Essential Critical Care Skills. www.baccn.org.uk/news/121105_National_Competency_Framework_for_Critical_Care_Nurse_Additional.doc (Last accessed: 18 April 2013. )

Leditschke I, Green M, Irvine J et al (2012) What are the barriers to mobilizing intensive care patients? Cardiopulmonary Physical Therapy Journal. 23, 1, 26-29.

Lemire F (2013) Competence, confidence, or both? Canadian Family Physician. 59, 3, 320.

McIntosh D, Spilka B (1990) Religion and physical health: the role of personal faith and control beliefs. Research in the Social Scientific Study of Religion. 2, 1, 167-194.

McLeod S (2009) Defense Mechanisms. www.simplypsychology.org/defense-mechanisms.html (Last accessed: 27 April 2013.)

Morris P, Herridge M (2007) Early intensive care unit mobility: future directions. Critical Care Clinician. 23, 1, 97-110.

Mudimela S (2010) Impact of level of participation on aggression, anxiety, achievement motivation and performance among soccer players. British Journal of Sports Medicine. 44, 1, 61.

National Institute for Health and Care Excellence (2009) Clinical Guideline 83: Rehabilitation after Critical Illness. National Institute for Health and Clinical Excellence: London.

Nauert R (2007) How Fear is Learned. http://psychcentral.com/news/2007/03/16/how-fear-is-learned/691.html (Last accessed: 17 April 2013.)

Newell B, Shanks D (2014) Unconscious influences on decision making: critical review. Behavioural and Brain Sciences. 37, 1-61.

Panksepp J (2010) Affective neuroscience of the emotional BrainMind: evolutionary perspectives and implications for understanding depression. Dialogues in Clinical Neuroscience. 12, 4, 533-545.

Perme C, Chandrashekar R (2009) Early mobility and walking program for patients in intensive care units: creating a standard of care. American Journal of Critical Care. 18, 3, 212-221.

Royal College of Nursing (2003) Guidance for Nurse Staffing in Critical Care. www.rcn.org.uk/_data/assets/pdf_file/0008/78560/001976.pdf. (Last accessed: 20 April 2017.) 
Schweickert W, Pohlman M, Pohlman A et al (2009) Early physical and occupational therapy in mechanically ventilated critically ill patients: a randomised controlled trial. The Lancet. 373, 9678, 1874-1882.

Smith J (2004) Reflecting on the development of interpretative phenomenological analysis and its contribution to qualitative psychology. Qualitative Research in Psychology. 1, 1, 39-54.

Smythe E, Ironside P, Sims S et al (2008) Doing Heideggerian hermeneutic research: a discussion paper. International Journal of Nursing Studies. $45,9,1389-1397$.

Stiller K (2007) Safety issues that should be considered when mobilizing critically ill patients. Critical Care Clinician. 23, 1, 35-53.

Truong A, Fan E, Brower R et al (2009) Bench-to-bedside review: mobilizing patients in the intensive care unit-from pathophysiology to clinical trials. Critical Care. 13, 216, 1-8.

Vincent J-L (2013) Critical care - where have we been and where are we going? Critical Care. 17, 1, 1-6.

West M, Borrill C, Dawson J et al (2002) The link between the management of employees and patient mortality in acute hospitals. International Journal of Human Resource Management.13, 8, 1299-1310.

Zomorodi M, Topley D, McAnaw M (2012) Developing a mobility protocol for early mobilization of patients in a surgical/trauma ICU. Critical Care Research and Practice. 1, 1-10. 